\title{
Selective visual-spatial attention alters induced gamma band responses in the human EEG
}

\author{
Thomas Gruber*, Matthias M. Müller, Andreas Keil, Thomas Elbert \\ Department of Psychology, University of Konstanz, PO Box D-25, D-78457 Konstanz, Germany
}

Accepted 6 July 1999

\begin{abstract}
Objectives: The present study was designed to investigate the attentional modulation of gamma band responses in a visual spatial attention task using a 128-channel-EEG-montage.

Methods: Colored rectangles were presented on a screen. After $500 \mathrm{~ms}$ an arrow indicated whether subjects had to shift their attention to the left or right half of the screen to detect target stimuli. During the task, either the attended half of the screen rotated horizontally while the unattended part remained motionless, or vice versa.

Results: When subjects attended the rotating stimulus, we found significantly higher power in a specific gamma band from $35-51 \mathrm{~Hz}$ on parieto-occipital electrode sites contralateral to the stimulation side. In addition, after the onset of the arrow which indicated what side subjects should direct their attention to, the $35-51 \mathrm{~Hz}$ response shifted from a broad posterior distribution to an increase of power at parietooccipital sites contralateral to the to-be-attended side. Furthermore, the rotating stimulus elicited higher gamma band power as compared to the standing stimulus at electrode locations, which may be related to the activity of underlying cortical structures specialized for motion processing.
\end{abstract}

Conclusions: The present results replicate important parts of previous findings of enhanced gamma power when a moving stimulus was attended.

Keywords: Human high density EEG; Induced gamma band response; Spatial attention; Perception; Vision

\section{Introduction}

The visual system is regarded as a hierarchically organized processing device, i.e. information is analyzed in multiple stages (Felleman and van Essen, 1991). The retinal image is gradually transformed from localized, spatially restricted representations in the striate cortex (area V1) to more global areas such as the middle temporal area (MT), specialized for the analysis of motion or area V4 which has been reported to contain color-selective neurons (Livingstone and Hubel, 1988; Zeki, 1993; Tootell et al., 1996). It was suggested that distinct regions of information processing remain segregated throughout higher areas in the brain and can be divided into two streams of information processing (Ungerleider and Haxby, 1994; Ungerleider, 1995). The occipito-temporal or ventral pathway is thought to be specialized for the analysis of object features (color, shape etc. - the 'what' system), and the occipito-parietal or dorsal pathway seems to be specialized for the analysis of motion

\footnotetext{
* Corresponding author.

E-mail address: thomas.gruber@uni-konstanz.de (T. Gruber)
}

and the spatial relationship between objects (the 'where' system; Ungerleider and Haxby, 1994).

Due to the finite computational resources of the brain, only a limited number of stimuli will be processed at a given time (Desimone and Duncan, 1995). Therefore it was suggested that a selective attention mechanism focuses these resources to specific objects or locations in the visual field without moving the eyes to that location (Hillyard et al., 1995; LaBerge, 1995; Hillyard and Anllo-Vento, 1998). Several metaphors have been used to explain this mechanism. It has been likened to a 'spotlight' (Posner et al., 1980; Posner and Petersen, 1990) or a 'zoom lens' (Eriksen and St. James, 1986).

In her 'feature integration theory', Treisman (Treisman and Gelade, 1980; Treisman and Gormican, 1988; Treisman, 1993) proposed that the visual system decomposes the visual input into maps of simple features such as color, shape and orientation, whereby only features within the spotlight of attention are combined to a coherent representation of the object (Treisman and Gelade, 1980). Psychophysiological studies measuring visual event-related potentials (VEPs) have shown that focusing the spotlight on 
a certain location in space leads to an enhancement of early sensory evoked responses as compared to when stimuli were presented outside the beam of the spotlight (e.g. Mangun et al., 1993; Gomez-Gonzales et al., 1994; Mangun, 1995; Anllo-Vento and Hillyard, 1996; Hillyard and AnlloVento, 1998; Hillyard et al., 1998; Luck and Ford, 1998). Moreover, human positron emission tomography (PET) studies have suggested that attending to a particular stimulus enhances cerebral blood flow in those cortical areas which are specialized for processing the relevant features of that stimulus (Corbetta et al., 1990; Corbetta et al., 1993; Heinze et al., 1994). Using functional magnetic resonance imaging (fMRI), it has been shown that metabolic activity in the parieto-occipital stream was enhanced when subjects attended to moving stimuli as compared to when the identical stimulus was ignored (Beauchamp et al., 1997; Haug et al., 1998). Thus, attention seems to have the effect of boosting or facilitating the activity of neurons in cortical areas or pathways which are related to processing the attended stimulus (Posner et al., 1980; Posner and Dehaene, 1994; LaBerge, 1995; Duncan et al., 1997; Luck et al., 1997; Hillyard et al., 1998).

However, all these findings have difficulties in explaining how a visual object is represented in the brain. One suggestion was - based on theoretical considerations and findings in intracortical recordings in animals - that the object is coded in a Hebbian-like neuronal cell assembly which is distributed across different functional visual areas by means of synchronized bursts of action potentials in a frequency range above $20 \mathrm{~Hz}$, i.e. the gamma band (Milner, 1974; Malsburg and Schneider, 1986;Eckhorn et al., 1990; Gray et al., 1990; Singer et al., 1990; Eckhorn et al., 1992; Singer and Gray, 1995). Contrary to the above mentioned VEPs, these synchronized oscillations are neither phase- nor time-locked to stimulus onset.

In human EEG, gamma band responses were induced by coherently moving lines giving the impression of a waterfall (Lutzenberger et al., 1995), by the short presentation of illusory Kanizsa and real triangles (Tallon et al., 1995; Tallon-Baudry et al., 1997b), by a visual search task (Tallon-Baudry et al., 1997a), by a coherently moving long bar (Müller et al., 1996,1997a,b) and by the perception of a Gestalt (Keil et al., 1999). In addition, induced gamma band responses were linked to cognitive processes, such as the processing of words (Pulvermüller, 1996; Pulvermüller et al., 1996, 1997), language and non-language stimuli (Eulitz et al., 1996) and, recently, to short-term memory processes during a delayed matching-to-sample task (Tallon-Baudry et al., 1998). All theses studies seem to support the idea of synchronized activity in wide spread cell assemblies linked to perceptual integration processes. However, in most of the studies, differences in induced gamma band activity were linked to the features of a stimulus, thus, stimuli which induced these activities were physically different from those which were linked to less power in the gamma band.
An alternative explanation for the findings in such experimental setups would be that the enhanced power in the gamma band is simply a by-product of stimulus processing (Kirschfeld, 1992). One method refuting this explanation is to employ an experimental setup which uses identical stimulus configurations. In one experimental condition, Tallon-Baudry et al. (1997a) presented their subjects an apparently meaningless picture that contained a hidden Dalmatian. The authors showed that gamma band power increased significantly when subjects perceived the dog as compared to when they viewed the picture without perceiving the dog. Here, it seems difficult to argue that the increase in gamma power is due to different features of the stimulus; in both cases the stimulus was identical with the exception that the dog was either perceived or unperceived. The limitation of this design is that once subjects perceive the dog, they will always perceive it. A method overcoming this limitations entails manipulating spatial selective attention. As mentioned above, early components of the VEP show a marked enhancement of amplitude when they were evoked by an attended stimulus as compared to when the identical stimulus was unattended (Mangun et al., 1993; Gomez-Gonzales et al., 1994; Mangun, 1995; Anllo-Vento and Hillyard, 1996; Hillyard and Anllo-Vento, 1998; Hillyard et al., 1998; Luck and Ford, 1998). This effect is linked to an amplification of neural responses in those visual pathways related to the processing of the stimulus (Hillyard et al., 1998). Consequently, one can hypothesize that if induced gamma band activity is a neural index of visual information processing, gamma power should be altered upon selective visual spatial attention by an amplification of the synchronized neural activity in the wide spread Hebbian cell assembly representing or coding the attended stimulus.

Previous studies on animals (Rougeul-Buser and Buser, 1994) and humans (Sheer, 1989) have suggested such an increase of gamma power is due to attentional processes. However, the experimental setup and the results of theses studies do not preclude that a shift of general arousal has caused the effects. Recently, we have demonstrated that power in a specific gamma band increased significantly when subjects attended to a long, coherently moving bar as compared to when that bar was ignored (Müller, 1998). Aside from behavioral data, we used objective physiological measures to control for subject's compliance and level of arousal. In particular, we were able to demonstrate that no differences between the experimental conditions were found with respect to alpha power, indicating no difference in the general level of arousal (Ota et al., 1996). We concluded that the increase in gamma power resulted from the amplification of synchronized neural spike activity in those cortical areas related to the processing of the moving bar (Müller, 1998). However, since we only recorded from a few occipital electrode sites, no information was obtained on the topography of that attention effect. In addition, aside from the standing bar serving as a baseline measure, no 
further standing control condition was introduced in order to compare the topographies for further strengthening of our conclusion of enhanced activity in the dorsal visual pathway when the bar was attended.

The present study was designed to overcome the limitations of our previous study by using a 128 channel EEGmontage and having not only a moving but also a standing control stimulus. Besides an enhancement in gamma power when the moving stimulus was attended, we expected an augmentation of gamma power on electrodes supposedly situated over cortical areas related to the dorsal stream in the moving as compared to the standing stimulus. In addition, we investigated whether a shift can be observed in the topographical distribution of the gamma band response to the hemisphere contralateral to the to-be-attended side after the onset of a attention direction cue. Thus, the present study goes beyond a simple replication of the effects found in our previous study.

\section{Materials and methods}

\subsection{Subjects}

Fourteen healthy, right-handed university students (6 males, 8 females), 22-30 years of age (mean $=25.8$ years, SD 2.7 years), participated in the experiment. All had normal or corrected-to-normal visual acuity. They received class credit or a financial bonus for participation. Informed consent was obtained from each participant.

\subsection{Stimuli and electrophysiological recordings}

As in our previous study (Müller, 1998) we used a moving-bar-like paradigm to induce gamma band activity. However, the paradigm was modified in that a standing stimulus control condition was added. Furthermore, in order to cover an identical visual field in the moving and standing condition, we used a complex pattern of squares and rectangles instead of a single bar. Stimuli were presented on a computer screen placed $1.5 \mathrm{~m}$ in front of the subjects with a frame rate of $70 \mathrm{~Hz}$. A red fixation square $0.4 \times 0.4^{\circ}$ was always present at the center of the screen. Each trial began with the presentation of the black screen for a period of $500 \mathrm{~ms}$. This epoch served as the baseline. After that, colored squares and rectangles were displayed on the screen for the following $500 \mathrm{~ms}$ (see Fig. 1A). The stimuli extended a visual angle of $4.7 \times 6.8^{\circ}$ from the fixation square for the left and right half of the screen, respectively. This period was introduced to measure the induced gamma band activity while subjects attended the whole screen in order to detect an arrow $\left(1.1 \times 1.1^{\circ}\right.$ due to the limited temporal resolution of fast Fourier transforms (FFTs) - we introduced a further period of $500 \mathrm{~ms}$ without the arrow before one side of the screen started to rotate horizontally (see Fig. 1C). The horizontal rotation $(2.6 \%$ s) was either on the left or right half of the screen and had a duration of 2000 ms. Thus, subjects either attended to the rotating or motionless screen, resulting in 4 experimental conditions (attend motion/unattended motion, left/right visual hemifield). With rotation onset, the squares and rectangles changed colors

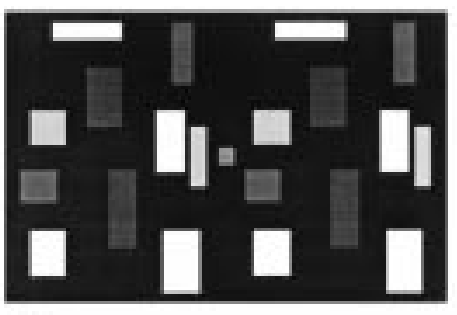

A

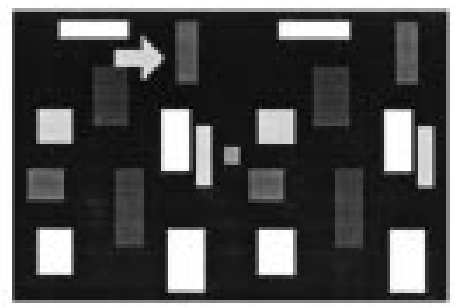

B

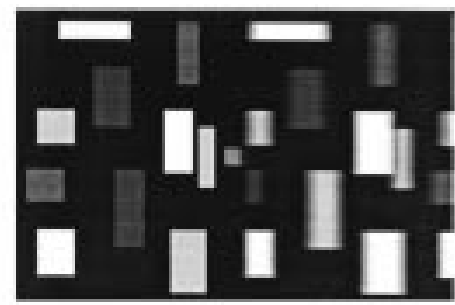

C

\begin{tabular}{c|c|c|c|c}
\hline $\begin{array}{c}\text { Baseline } \\
\text { (black screen) }\end{array}$ & $\begin{array}{c}\text { Stimulus } \\
\text { Onset }\end{array}$ & Arrow & $\begin{array}{c}\text { Standing Stimulus } \\
\text { without arrow }\end{array}$ & $\begin{array}{c}\text { Horizontal rotation } \\
\text { (left or right } \\
\text { half of the screen) }\end{array}$ \\
\hline $500 \mathrm{~ms}$ & $500 \mathrm{~ms}$ & $500 \mathrm{~ms}$ & $500 \mathrm{~ms}$ & $2000 \mathrm{~ms}$ \\
\hline $\mathrm{D}$
\end{tabular}

Fig. 1. Schematic representation of one trial. (A) Stimulus onset. (B) Arrow indicating whether the subject had to attend the left or the right half of the screen. (C) Rotation of the left or right half of the screen. (D) Schematic time course of one trial. 
independently on both the rotating and motionless halves of the screen 3-5 times per $2000 \mathrm{~ms}$. Among the color changes, one of the squares or rectangles unpredictably changed to the color brown. This color change was defined as the target and the subjects" task was to silently count the number of targets per trial. Within one trial, between 0 and 3 target presentations were possible. At the end of each trial, subjects had to enter how many targets they had counted via the numeric keyboard. Feedback was provided immediately on the screen. The direction of rotation was horizontal towards the center of the screen, since in pre-experimental test sessions we determined that this direction of rotation prevented subjects from following the rotation with their eyes.

The next trial started with a delay of $500 \mathrm{~ms}$ after subjects hit any key on the numeric keyboard. This delay was introduced in order to avoid contamination of the post motorpotential during the following $500 \mathrm{~ms}$ black screen baseline period (Neshige et al., 1988). A schematic time course for one trial is given in Fig. 1D. All events were synchronized to the vertical retrace of the monitor. Four hundred and eighty trials were presented in random order which resulted in 120 trials per experimental condition.

Subjects were instructed to avoid eye movements and blinks while attending the screen and accurately count the number of targets per trial. Before the beginning of electrophysiological recordings, subjects were trained to detect targets and the arrow without moving their eyes. Inter-trial intervals were dependent on subjects keyboard hits to start the new trial.

EEG was recorded continuously using a 128-channel Electrical Geodesic ${ }^{\mathrm{TM}}$ system with a sampling frequency of $250 \mathrm{~Hz}$ referenced to the vertex electrode. An online bandpass filter from 0.1 to $100 \mathrm{~Hz}$ was used. Impedances were kept below $50 \mathrm{k} \Omega$. In addition, vertical and horizontal eye movements were monitored with a subset of the 128 electrodes. Further data processing was performed off-line.

\subsection{Data reduction}

For frequency analysis, single epochs of $4000 \mathrm{~ms}$ length were extracted. Thus, one epoch consisted of $500 \mathrm{~ms}$ baseline (black screen), $500 \mathrm{~ms}$ stimulus presentation prior to the attention direction arrow, $500 \mathrm{~ms}$ with arrow on the screen, $500 \mathrm{~ms}$ without arrow, and $2000 \mathrm{~ms}$ of one half of the screen in rotation. These epochs were submitted for artifact rejection and correction according to a procedure developed by Junghöfer et al. (statistical correction of artifacts in dense array studies, SCADS, in press). This procedure (1) detects individual recording channel artifacts using the recording reference, (2) detects global artifacts using the average reference, (3) replaces sensors contaminated with artifacts with statistically-weighted spherical interpolation from the full channel set, and (4) computes the variance of the signal across trials to document the stability of the average waveform. The limit for the number of approximated channels was set to 20 channels. Single epochs with excessive eyemovements and blinks and more than 20 channels containing artifacts were totally excluded from further analysis. Using this method, 3 subjects were excluded due to excessive artifacts. For the remaining 11 subjects the average rejection rate was $30 \%$. For further analysis the average reference was used.

A given EEG-epoch can be modeled by the sum of the evoked response plus the trial-by-trial fluctuation around the mean (Priestley, 1988). Since the present analysis focused on non phase-locked oscillatory activity, the evoked response (i.e. the VEP) was subtracted from each trial (see also Müller et al., 1996, for a similar procedure). Spectral analysis was then calculated on artifact-free trials by means of a FFT algorithm developed by Feige (1996). A moving window FFT (overlapping Welch windows of $192 \mathrm{~ms}$ ) was performed for single trials resulting in a frequency resolution of $7.8 \mathrm{~Hz}$ (first frequency range $0-3.9 \mathrm{~Hz}$, then $7.8 \pm$ $3.9 \mathrm{~Hz}$ etc. to $117 \pm 3.9 \mathrm{~Hz}$ ). Time resolution i.e. the steps of shifting was $60 \mathrm{~ms}$ (see also Makeig, 1993, for a similar procedure). Single trial time by frequency matrices were then averaged for each electrode. Four frequency bands were formed in the gamma band range out of the averaged time by frequency matrices by averaging the respective frequencies $(19-35,35-51,51-66$ and $66-82 \mathrm{~Hz})$ and a lower frequency range from 4 to $12 \mathrm{~Hz}$. Because of the online $100 \mathrm{~Hz}$ lowpass filter, higher frequencies were not analyzed.

\subsection{Attention effect and attentional shift}

As an estimate of general noise, the mean power of the first $500 \mathrm{~ms}$ while the screen was black (no overlap with stimulus onset) was calculated for each frequency band. The time by frequency band matrices were divided by the mean baseline power. Thus, the normalized time windows represent the spectral power relative to the dark screen. To further reduce the data for statistical analysis, the following averages across time were calculated for each frequency band: (1) to test the general attention effect after motion onset, the mean baseline corrected power was calculated for the time period 44 to $1376 \mathrm{~ms}$ after motion onset (which is $1544-2876 \mathrm{~ms}$ after stimulus onset), (2) to track the shifting of attention, 3 time averages were computed (2a) from 44 to $476 \mathrm{~ms}$ after stimulus onset, (2b) from 524 to $956 \mathrm{~ms}$ after stimulus onset, which is the time period while the arrow was present, and, (2c) from 1064 to $1496 \mathrm{~ms}$ after stimulus onset, which is the time period between arrow offset and motion onset. Thus, all time averages represent non-overlapping epochs in the frequency domain.

With respect to spatial sampling points, electrodes were collapsed into 12 electrode-clusters according to 12 regional means proposed for 64 electrode sites by Dien et al. (1997) which are shown in Fig. 2.

As depicted in Fig. 2, this procedure resulted in 6 regional means for each hemisphere: frontal (1 and 7), anterior 


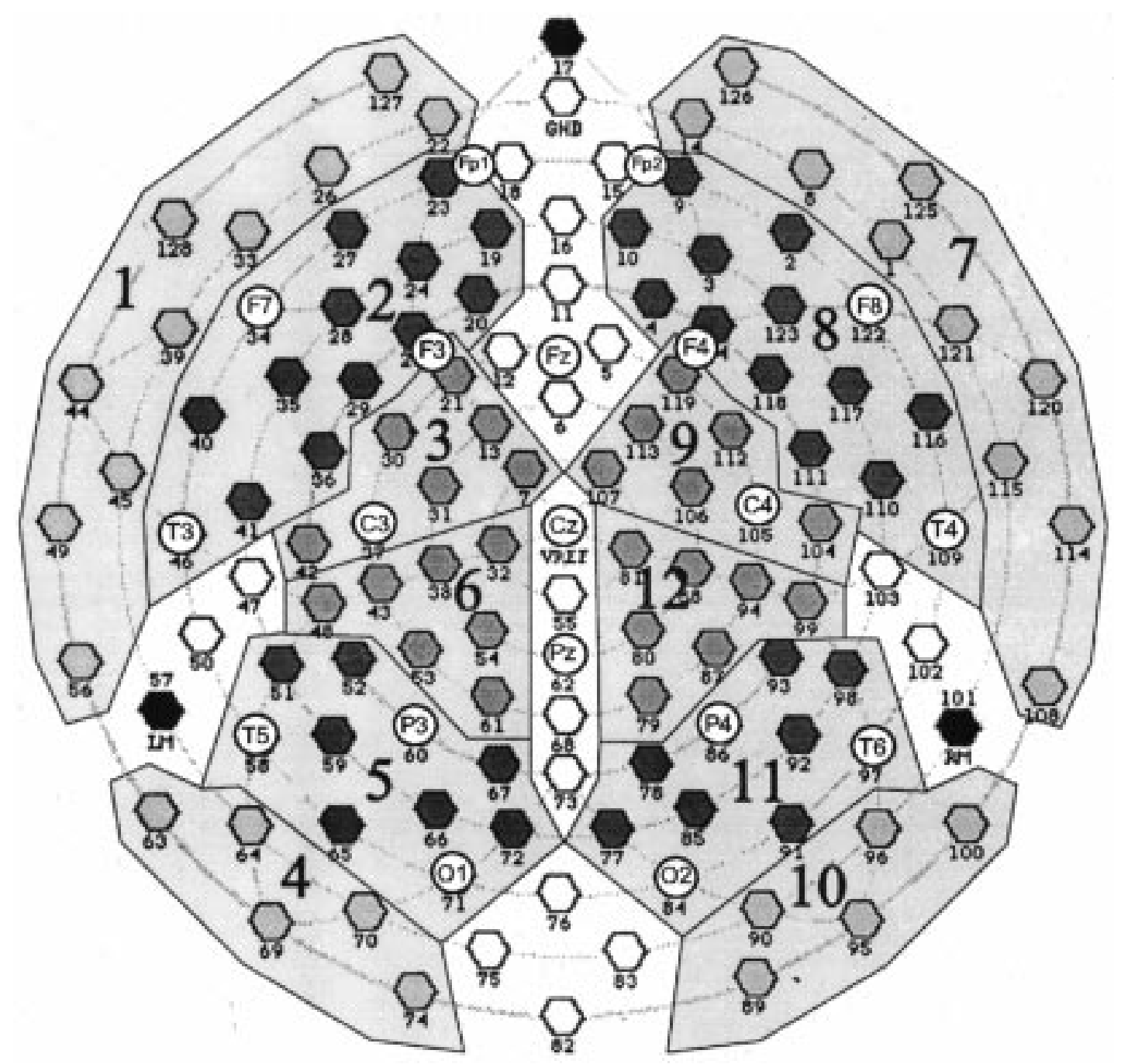

Fig. 2. Regional means resulting from the 128 channels montage. 10-20 electrode sites are given to allow for a comparison.

lateral ( 2 and 8 ), anterior superior (3 and 9), posterior superior (6 and 12), parieto-occipital (5 and 11), and posterior inferior (4 and 10). The averaged power across the respective electrode sites was calculated for these regional means for each frequency band.

\subsection{Motion related gamma power}

To test whether motion induces activity at specific electrode sites as compared to the standing stimulus, the frequency band exhibiting a significant attention effect was further analyzed with a slightly different procedure. Instead of using the black screen as the baseline period, here the mean spectral power from 1064 to 1496 ms after stimulus onset was used, i.e. the time period between arrow offset and motion onset. Thus, attention was shifted to the relevant visual hemifield but the screen was still motionless. For the statistical analysis, the mean power across time from 44 to $1376 \mathrm{~ms}$ after motion onset was calculated and divided by the above mentioned the time period for each of the relevant electrodes, respectively. In order to obtain the gamma band activity which is related to motion, we subtracted the normalized spectral power for the attended motionless half of the screen from the normalized power of the identical attended rotating half of the screen. This resulted in two measures: attend-left rotating minus standing screen and attend-right rotating minus standing screen. We introduced this difference measure in order to control, not only for the attention effect, but also for the color changes which defined targets and standards during the last $2 \mathrm{~s}$ of each trial.

Previous studies investigating motion related VEPs reported the most pronounced effects on parieto-occipital electrode sites P3/4 and O1/2 (e.g. Probst et al., 1993; Anllo-Vento and Hillyard, 1996; Patzwahl et al., 1996; Buchner et al., 1997; Valdes-Sosa et al., 1998). On the basis of these results, we calculated two regional means for electrodes 71, 66 and 60 for the left and 84, 85 and 86 
for the right hemisphere (see Fig. 2). These electrodes cover the area between P3/4 and O1/2 of the international 10-20 system, respectively (Fig. 2).

Topographies were calculated with an algorithm developed by Junghöfer (Junghöfer et al., 1997, 1999), which uses Perrin-like spherical spline interpolations.

\subsection{Statistical analysis}

For the evaluation of interactions including the regional means, the respective frequency band was normalized as proposed by McCarthy and Wood (1985) which required that, for each condition, the minimum and maximum power for each electrode and subject be determined and the normalized value $n$ at each electrode $j$ be computed according to equation

$n_{j}(t)=\frac{x_{j}(t)-\min }{\max -\min }$

$X j(t)$ : power of the sensor $j$ at time $t$.

\subsubsection{Attention effect}

In a first step, we tested whether attention to the rotating half of the screen induces increased activity in a specific gamma band at parieto-occipital electrode sites as compared to ignoring the motion in the same visual hemifield. Therefore, baseline corrected and normalized power of each of the 5 frequency bands over the left and right parieto-occipital regional means (5 and 11 in Fig. 2) averaged across time from 44-1376 ms after motion was tested by a 3-factor repeated measures analysis of variance (ANOVA; left/ right Regional mean $\times$ left/right Visual hemifield $\times$ attended/unattended rotation). In addition, a post-hoc repeated measures ANOVA was calculated for the difference between attended and unattended rotation (left/right regional mean $\times$ left/right visual hemifield) in order to analyze hemispherical asymmetries of the attention effect.

For testing the topographical distribution, the frequency band which exhibited a significant attention effect was tested by a 3-factor repeated measures ANOVA with all 12 regional means (regional mean $\times$ visual hemifield $X$ attended/unattended rotation). Post-hoc comparisons were evaluated by means of paired $t$ tests.

\subsubsection{Attentional shift}

We hypothesized that a shift of gamma band activity from a relatively broad distribution when attending the whole screen to a maximum over the hemisphere contralateral to the to-be-attended visual hemifield would occur in parietooccipital areas. Each of the 3 temporal means prior to motion onset (see above) for the frequency band which exhibited a significant increase while attending the rotating screen was tested by a 2-factor repeated measure ANOVA (Regional mean $\times$ attention - left/right visual hemifield). It was expected that only 2 temporal means, from arrow onset to motion onset, exhibit a significant regional mean by attention interaction.

\subsubsection{Motion related gamma power}

The motion related regional means (see above) were again tested by a 2-factor repeated measures ANOVA (left/right regional mean $\times$ attended visual hemifield) for the frequency band which exhibited a significant attention effect.

Where appropriate, degrees of freedom were adjusted by Huynh and Feldt epsilon in all ANOVA models. Means and standard errors are presented.

\section{Results}

\subsection{Behavioral data}

On average 708 targets were presented. Participants missed between 144 and 281 of the brown squares or rectangles $($ mean $=232.5, \mathrm{SD}=40.5)$, i.e. participants detected approximately $67 \%$ of target stimuli in each condition.

\subsection{Electrophysiological recordings}

\subsubsection{Attention effect}

Fig. 3 depicts the normalized mean spectral power relative to baseline for all 5 frequency bands across subjects and the left and right parieto-occipital regional means.

Overall, when the rotating screen was attended, only the frequency band between $35-51 \mathrm{~Hz}$ exhibited a significant power increase over the left and right parieto-occipital regional means as compared to the unattended rotation $(F(1,10)=8.64, P=0.01)$. This increase was higher at parieto-occipital sites contralateral to the to-be-attended rotating screen and more so when the rotation on the left visual hemifield was attended as can be seen in Fig. 4 (Regional mean $\times$ visual hemifield $\times$ attention, $F(1,10)=$ 6.87, $P<0.05$ ).

Post-hoc paired $t$ tests revealed a significant increase of

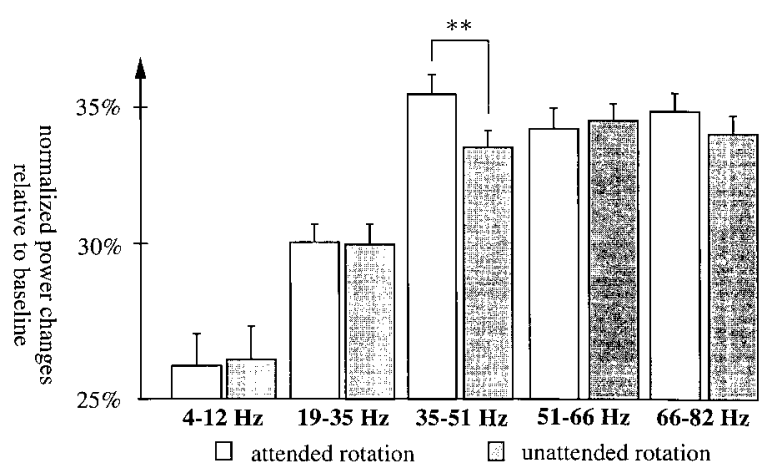

Fig. 3. Normalized power changes (+ standard error) across all subjects relative to baseline across the left and right parieto-occipital regional means when subjects attended (white bars) or ignored (grey bars) the rotating screen, respectively. ${ }^{* *} P \leq 0.01$. 


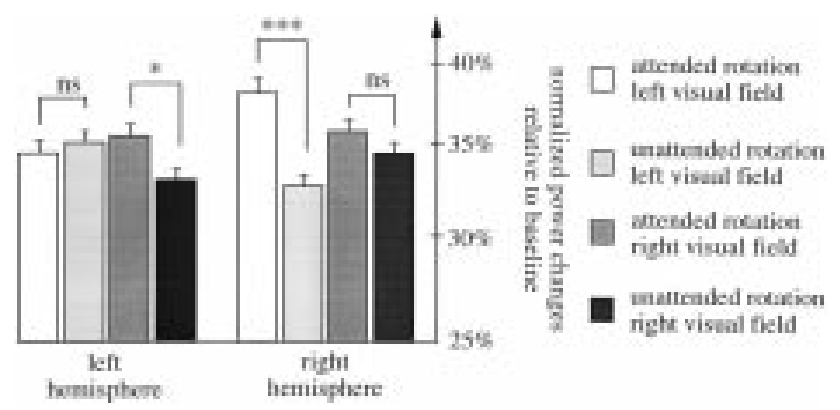

Fig. 4. Grand mean normalized spectral power (+ standard error) relative to baseline in the frequency range from $35-51 \mathrm{~Hz}$ at parieto-occipital regional means when subjects attended the left or right visual hemifield, respectively. ${ }^{* * *} P \leq 0.001 ; * P \leq 0.05$; ns, not significant.

power in the $35-51 \mathrm{~Hz}$ band over the right parieto-occipital region when motion was attended in the left visual hemifield as compared to when left visual hemifield motion was ignored $(t(10)=4.70, \quad P<0.001 ; \quad$ relative increase $21.2 \%)$. Over the left parieto-occipital region, the attended rotation in the right visual hemifield revealed a significant enhancement of gamma spectral power as compared to the ignored motion in the same visual hemifield $(t(10)=2.74$, $P<0.05$; relative increase $10.0 \%$ ). No significant differences in the frequency range from $35-51 \mathrm{~Hz}$ were found at regional means ipsilateral to the attended or unattended motion (left hemisphere: $t(10)=-0.41, P=0.69$; right hemisphere: $\quad t(10)=1.08, \quad P=0.30)$. The post-hoc ANOVA on the spectral power differences between the attended and unattended rotation revealed that activation was more pronounced for the right parieto-occipital regional mean $(F(1,10)=6.45, P<0.05)$.

Fig. 5A depicts the grand mean topographical distribution of the $35-51 \mathrm{~Hz}$ power for the attended and unattended motion of the left visual hemifield.

The examination of the topographies of the $35-51 \mathrm{~Hz}$ band over the whole scalp revealed a significant regional mean visual hemifield $\times$ attention interaction $(F(11,110)=$ $2.48, P<0.05$ ), which was expected due to the above reported results. However, not only the parieto-occipital but also the left and right frontal regional means (1 and 7 in Fig. 2) exhibited a marked increase in spectral power when motion was attended. As with the parieto-occipital sites, post-hoc t-tests resulted in a significant enhancement of gamma band power over the left frontal area when motion in the left visual hemifield was attended as compared to when motion was ignored in the same visual hemifield $(t(10)=2.60, P<0.05)$. The same was true for right frontal areas with respect to the right visual hemifield $(t(10)=2.4, P<0.05)$. Furthermore, general power across attended and unattended motion at parieto-occipital regional means was significantly higher as compared to posterior-inferior $(t(10)=19.55, P<0.0001)$ and posteriorsuperior $(t(10)=12.82, \quad P<0.0001)$ regional means (Fig. 6).

\subsubsection{Attentional shift}

Fig. 7 depicts the mean baseline and normalized spectral power for the $35-51 \mathrm{~Hz}$ band across subjects for the 3 time averages prior to motion onset for the left and right parietooccipital sites when attention was shifted to either the right or left visual hemifield.

As can be seen in Fig. 7, spectral power increased in the second and third time average at the parieto-occipital regional mean contralateral to the to-be-attended visual hemifield. These two time averages represent the period during direction arrow onset to motion onset. Furthermore, the ipsilateral parieto-occipital spectral power showed only marginal differences as compared to the first time average in which the whole screen was attended. Consequently, we found a significant regional mean $\times$ attention interaction for time average two $(F(3,30)=4.30, P<0.05)$ and three $(F(3,30)=6.46, P<0.01)$ only. In Fig. 5B, the topographies for the time period while the subjects are waiting for the arrow to occur and - in this example - when the shift to the left visual hemifield has taken place are depicted. From a relatively broad gamma band distribution, spectral power was lateralized or shifted towards the contralateral hemisphere after the arrow was present. Interestingly, as can be seen from Fig. 5B and 7, there is no indication of a power suppression at the ipsilateral hemisphere when attention was shifted to either the left or right visual hemifield. It seems as if attention produces an amplification of power over electrodes sites contralateral to the attended stimulus side only.

\subsubsection{Motion related gamma power}

Here we tested whether motion, i.e. rotation, is related to an increase of spectral power in comparison to a motionless stimulus at electrode sites supposedly linked to measuring cortical activity related to motion processing (see Section 2). The power difference (attended-rotating minus attendedmotionless half of the screen) which was normalized to the time period after the shift of attention had already taken place revealed a significant increase in power in the 35-51 $\mathrm{Hz}$ band at those electrodes which were contralateral to the attended visual hemifield $(F(1,10)=5.11, P<0.05)$. Rotation resulted in an power increase of $9.7 \%$ for the left and of $9.6 \%$ for the right visual hemifield at those electrodes which covered the scalp region of $\mathrm{P} 3 / 4$ to $\mathrm{O} 1 / 2$ of the international 10-20 system. The grand mean topographical distribution across 11 subjects is depicted in Fig. 5C for the left visual hemifield.

\section{Discussion}

The purpose of the present study assisted in addressing the following questions: (1) can we replicate our previous finding of an enhanced spectral gamma band power when a moving stimulus was attended as compared to when the identical stimulus was ignored (Müller, 1998)? (2) Is the shift of visual spatial attention to either the left or right 

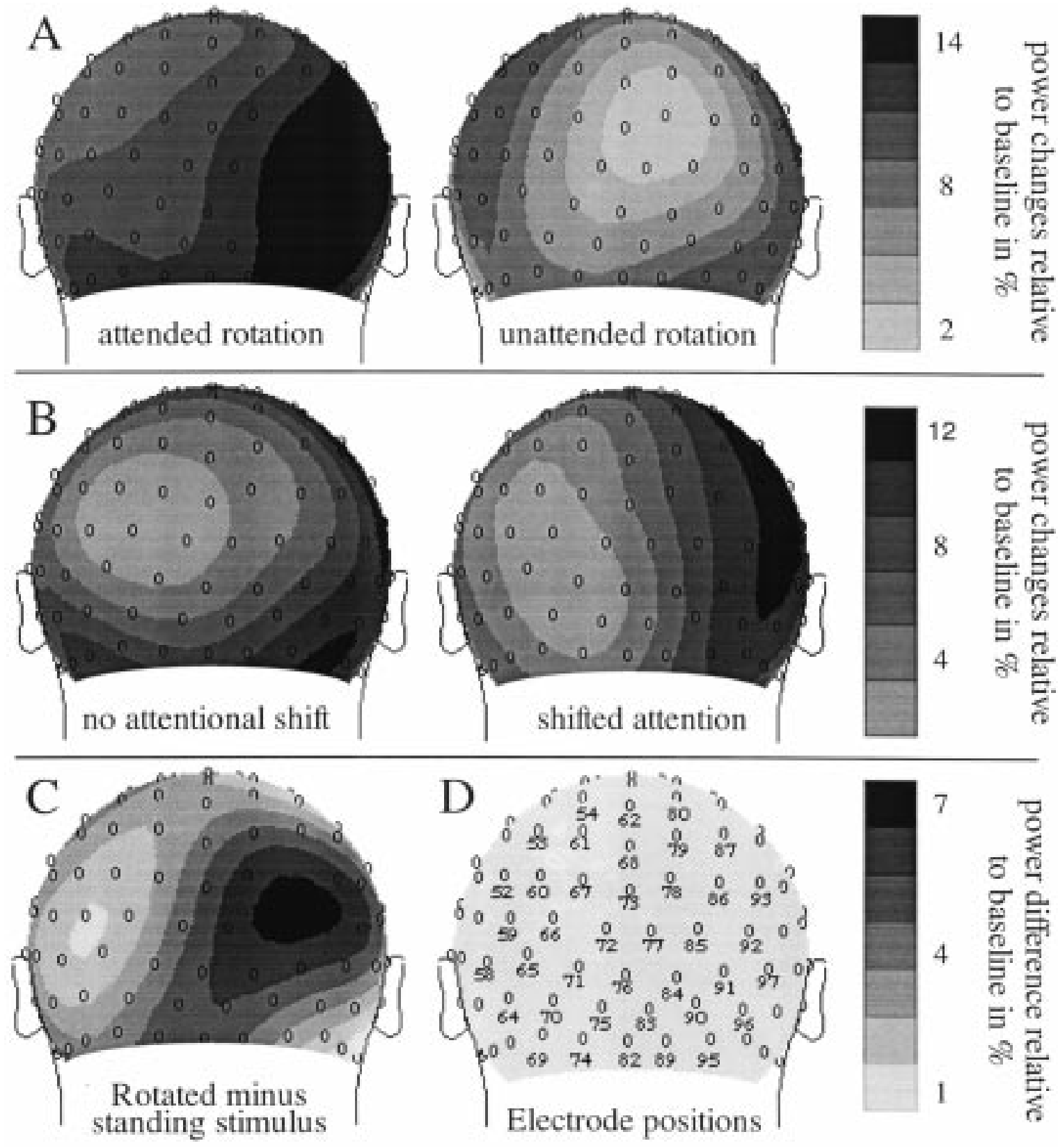

Fig. 5. Spherical spline topographical distributions of the $35-51 \mathrm{~Hz}$ band across 11 subjects. (A) Attended and unattended rotation in the left visual hemifield (44-1376 ms after motion onset) relative to baseline (black screen). (B) 44-476 ms after stimulus onset, i.e. no attentional shift and 1064-1496 ms after stimulus onset, i.e. shifted attention (to the left visual hemifield) relative to baseline (black screen). (C) Rotated minus motionless stimulus in the left visual field (44-1376 ms after motion onset) relative to spectral power before motion onset. (D) Posterior electrode positions.

visual hemifield accompanied by a shift of gamma power towards the contralateral cortical hemisphere? (3) Is the attentional enhancement of spectral power related to the processing of motion, i.e. resulting from an amplification of neural synchronized activity in the dorsal pathway as suggested by studies using motion evoked VEPs (AnlloVento and Hillyard, 1996; Valdes-Sosa et al., 1998)? A 128-channels EEG montage was used in order to reveal high-density spatial information.

Results demonstrated a replication of our previous findings of an attentional alteration of induced gamma band responses by selective visual-spatial attention. However, contrary to our preceding experiment using a single long moving bar, in which we found an augmentation in the $65-96 \mathrm{~Hz}$ band, the present experiment resulted in an augmentation of spectral gamma power in a frequency range from $35-51 \mathrm{~Hz}$. It is currently unclear as to what accounts for the differences in the reported frequency ranges across human gamma band studies. An increase of gamma power was reported for coherently moving lines giving the impression of a waterfall at 35-45 $\mathrm{Hz}$ (Lutzenberger et al., 1995), for a coherently moving long bar at $40-96 \mathrm{~Hz}$ 


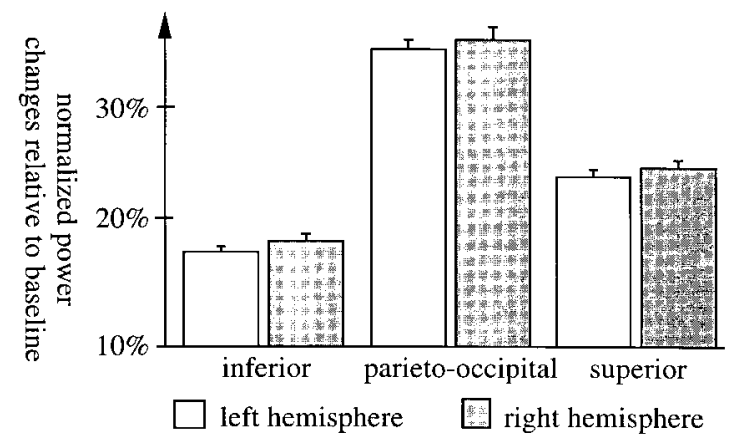

Fig. 6. Normalized grand mean spectral power $(+$ standard error $)$ in a frequency range from $35-51 \mathrm{~Hz}$ relative to baseline at 6 posterior regional means for time window 44-1376 ms after motion onset, averaged across all 4 conditions, i.e. attended and unattended rotation in the left or right visual hemifield.

(Müller et al., 1996) which was replicated for the same frequency band (Müller et al., 1997b), for a visual search task around $40 \mathrm{~Hz}$ (Tallon-Baudry et al., 1997a), after the short presentation of illusionary and real triangles at $30 \mathrm{~Hz}$ (Tallon et al., 1995) which was replicated at $40 \mathrm{~Hz}$ (TallonBaudry et al., 1996, 1997b) and, recently, for a delayedmatching-to-sample task at $24-60 \mathrm{~Hz}$ (Tallon-Baudry et al., 1998). It seems likely that both the variance in tasks and stimulation account for the variety of results. Here, we used a more complex stimulation material than in our preceding experiment. This might contribute to our finding of an attention effect in a lower frequency range. In addition, it should be mentioned that in the present study we were not able to analyze frequencies higher than $85 \mathrm{~Hz}$ due to technical limitations.

In our previous study, we used a $3 \times 3$ occipital electrode array which allowed only limited conclusions with regard to the topographical distribution. With the high-density 128 electrodes array of the present study, we found significantly higher gamma power on parieto-occipital electrode sites contralateral to the attended rotating half-screen as compared to when the identical half of the screen was ignored. However, this effect was more pronounced when the left visual hemifield was attended. Such a hemispheric asymmetry has been frequently reported in spatial visual attention tasks using VEPs (Palmer and Tzeng, 1990; Posner and Petersen, 1990; Posner and Dehaene, 1994; Heilman, 1995) and PET (Corbetta et al., 1993, 1995). In their study, Corbetta et al. (1993) reported an asymmetrical activation of the parietal cortex when attending the left or right visual field. Attention to the left visual field was mostly controlled by one region in the right parietal cortex, while attention to the right field was controlled more bilaterally, by a left parietal and a distinct right parietal region.

In addition to the parieto-occipital maximum, we found a second maximum at frontal regional means. Here we can only speculate whether this activity is related to the anterior attention system (Posner et al., 1987; Posner and Petersen, 1990; Posner and Dehaene, 1994) or whether these anterior maxima resulted from the projection of a posterior dipole. Recently, Corbetta and Shulman (Corbetta and Shulman, 1998) found that covert shifting of attention to a certain location of the visual field was related to an increase of blood flow in frontal areas such as the frontal eye field and the supplementary eye field. The authors concluded that a fronto-parietal network may be responsible for the spatial attentional modulations in the ventral visual system. Our present findings may well suit a model of a synchronized fronto-parietal network.

The second aim of the present study was to investigate whether the attentional shift to either the left or right visual hemifield is related to a topographical shift of gamma power to the respective contralateral hemisphere. In the time period during which subjects had to attend the whole screen in order to detect the attention direction arrow, we saw a broad posterior distribution of spectral power in the 35-51 $\mathrm{Hz}$ band. Such a broad distribution would fit Vin's (Vijn, 1992; Vijn et al., 1992) model of the visual cortex being in a 'scanning state'. While the visual system is scanning the environment for relevant information, many visual areas are involved and should be linked together in order to allow for a rapid discrimination of new, relevant visual information. After the arrow appeared on the screen, we observed an increase of gamma power over the side contralateral to the to-be-attended hemisphere. We found no significant reduction of gamma power over the ipsilateral hemisphere, giving rise to the notion of a facilitation of those visual areas which are related to the information processing of the attended visual hemifield. These findings are in accordance with a recent study which reported a cortical facilitation during cued shifts of spatial attention using the steady-state visual evoked potential (SSVEP; Müller et al., 1998). When two flickering stimuli were presented in the left and right visual hemifield, the shift of attention to one of the stimuli resulted in an increase of SSVEP amplitude for the attended side with no signs of a

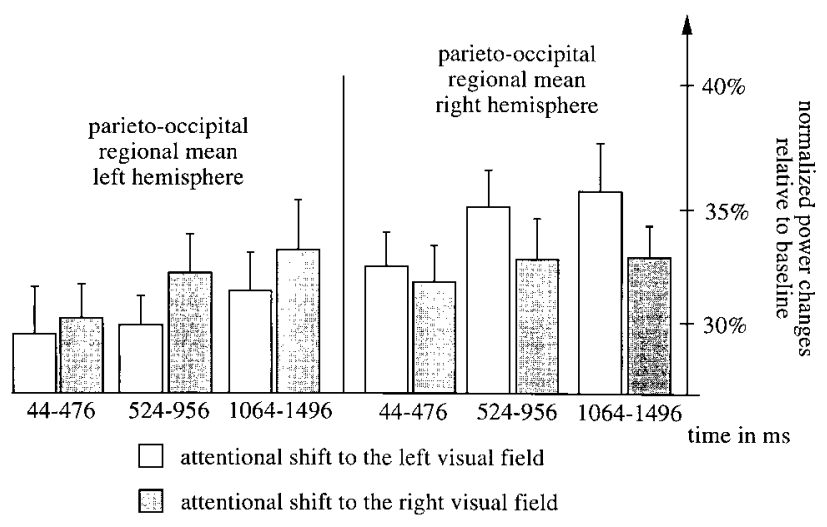

Fig. 7. Time course of grand mean normalized spectral $35-51 \mathrm{~Hz}$ power ( + standard error) relative to baseline for parieto-occipital regional means for the first three $500 \mathrm{~ms}$ epochs after stimulus onset when subjects had to shift their attention to the left (white bars) or right (grey bars) visual hemifield, respectively. Note: time is given in the time domain. 
SSVEP amplitude reduction for the ignored stimulation side. We proposed that the observed facilitation represents the operation of a sensory gain-control mechanism that enhances the signal-to-noise ratio and hence the discriminability of those stimuli which are at the attended location (Hillyard et al., 1998; Müller et al., 1998).

The third aim of the present study was to test whether the increase in gamma band power while attending a moving stimuli is related to a facilitation of the dorsal visual pathway (Ungerleider and Haxby, 1994; Ungerleider, 1995). Previous studies which investigated the motion VEP reported an increase in amplitude at electrode sites P3/4 and $\mathrm{O} 1 / 2$ of the international 10-20 system when the moving stimulus was attended and related this augmentation to activity in the dorsal stream (Hillyard et al., 1998; Müller et al., 1998; Valdes-Sosa et al., 1998). Human fMRI studies showed an increase of metabolic activity related to the processing of moving stimuli in a cortical region which underlies approximately that area measured by P3/4 and O1/2 (Tootell et al., 1995, 1996; Sereno et al., 1995). We demonstrated a significant increase of gamma band activity at regional means covering the area of electrode positions P3/4 to O1/2 when the rotating screen was attended relative to when the standing screen was attended. Our results are in line with human fMRI findings from Beauchamp et al. (1997); Haug et al. (1998), who demonstrated an increase in metabolic activity in the middle temporal area (MT) when a moving stimuli was attended. This increase in activation might result from a larger electrocortical response.

It has been suggested to calculate the current source density (CSD, Perrin et al., 1989; Nunez, 1990) before the transformation into the frequency domain, in order to get a reference free measure of cortical activity (Lutzenberger et al., 1995; Pulvermüller et al., 1997). However, the CSD seems not to be suitable for deep or distributed sources, since it emphasizes sharp features in the scalp topography (Dien, 1998). In addition, calculating the CSD for single trials bury the problem of overestimating general noise and may also introduce high-frequency noise to the temporal wave form (Biggins et al., 1991; Law et al., 1993). Such high-frequency noise is particularly problematic when analyzing data in the frequency domain, and more so, when one is interested in the gamma band frequency range. On the basis of simulated data it was argued that the average reference may produce so-called 'ghost fields' (Desmedt et al., 1990) and can produce distortions of focal features (Tomberg et al., 1990). However, as Dien (1998) argued, such problems mainly occur when using sparse electrode arrays. He recommended to use the average reference for deep or distributed sources in combination with high density electrode arrays (Dien, 1998). In the present study we expected rather distributed sources and were interested in the gamma frequency range, thus we decided to analyze our data based on the average reference.

We can not completely rule out that muscle activity has contributed to the findings in the gamma band range.
However, no attentional modulation of gamma band spectral power was found at regional means closest to the neck muscles (see posterior-inferior regional means 4 and 10 in Fig. 2). In addition, the attention effect was most prominent on electrodes contralateral to the to-be-attended visual hemifield, whereas neck muscle activity should result in a more symmetrical pattern. Given the random attentional cueing in the present study, it does not seem plausible that subjects have systematically tensed right head muscles when shifting their attention to the left visual hemifield and vice versa. Furthermore, muscle activity shows its peak amplitude well beyond $60 \mathrm{~Hz}$ (Pulvermüller et al., 1997), which is above the $35-51 \mathrm{~Hz}$ band.

Regarding gamma activation at anterior electrode positions, it may be asked whether eye movements have contributed to the present findings. We addressed this issue by examining EOG activity extensively for artifacts produced by eye movements or blinks. In addition, one might expect a bilateral topographical distribution of EOG activity independent of the experimental condition.

In summary, the present experiment replicated previous findings of a power enhancement when a moving stimulus is attended. This enhancement was most prominent at parietooccipital electrodes contralateral to the to-be-attended visual hemifield and it seems plausible that this enhancement resulted from an amplification of synchronized neural activity in the visual dorsal pathway. Shifting visual attention to one visual hemifield was related to a power amplification over the contralateral hemisphere with no sign of a power suppression over the ipsilateral hemisphere. Results strongly suggest that induced gamma band activity is closely related to visual information processing and attentional perceptual mechanisms.

\section{Acknowledgements}

We are grateful to Ursula Lommen and Jürgen Wolf for help in data acquisition and to Lisa Green for editorial support. Research was supported by grants from the Deutsche Forschungsgemeinschaft and the Human Frontier Science rogram.

\section{References}

Anllo-Vento L, Hillyard SA. Selective attention to the color and direction of moving stimuli: electropysiological correlates of hierarchical feature selection. Perception Psychophysics 1996;58:191-206.

Beauchamp MS, Cox RW, DeYoe EA. Graded effects of spatial and featural attention on human area MT and associated motion processing areas. J Neurophysiol 1997;78:516-520.

Biggins EA, Fein G, Rax J, Amir A. Artifactually high coherences from using spherical spline computation of scalp current density. Electroenceph clin Neurophysiol 1991;79:413-419.

Buchner H, Gobbele R, Wagner M, Fuchs M, Waberski TD, Beckmann R. Fast visual evoked potential input into human area V5. NeuroReport 1997;8:2419-2422.

Corbetta M, Shulman GL. Human cortical mechanisms of visual attention 
during orienting and search. Philos Trans R Soc Lond B Biol Sci 1998;353:1353-1362.

Corbetta M, Miezin FM, Dobmeyer S, Shulman GL, Petersen SE. Attentional modulation of neural processing of shape, color, and velocity in humans. Science 1990;248:1556-1559.

Corbetta M, Miezin FM, Shulman GL, Petersen SE. A PET study of visuospatial attention. J Neurosci 1993;13:1202-1226.

Corbetta M, Shulman GL, Miezin FM, Petersen SE. Superior parietal cortex activation during spatial attention shifts and visual feature conjunction. Science 1995;270:802-805

Desimone R, Duncan J. Neural mechanisms of selective visual attention. Ann Rev Neurosci 1995;18:193-222.

Desmedt JE, Chalkin V, Tomberg C. Emulation of somatosensory evoked potential (SEP) components with the 3-shell head model and the problem of 'ghost potential fields' when using an average reference in brain mapping. Electroenceph clin Neurophysiol 1990;77:243-258.

Dien J. Issues in the application of the average reference: review, critiques, and recommendations. Behavior, Research Methods, Instruments and Computers 1998;30:34-43.

Dien J, Tucker DM, Potts G, Hartry-Speiser A. Localization of auditory evoked potentials related to selective intermodal attention. J Cog Neurosci 1997;9:799-823.

Duncan J, Humphreys G, Ward R. Competitive brain activity in visual attention. Curr Opin Neurobiol 1997;7:255-261.

Eckhorn R, Reitboeck HJ, Arndt M, Dicke P. Feature linking via synchronization among distributed assemblies: simulations of results from cat visual cortex. Neural Computation 1990;2:293-307.

Eckhorn R, Schanze T, Brosch M, Salem W, Bauer R. Stimulus-specific synchronizations in cat visual cortex: multiple microelectrode and correlation studies from several cortical areas. In: Basar E, Bullock TH, editors. Induced rhythms in the brain, 1992. pp. 47-82.

Eriksen CW, St. James JD. Visual attention within and around the field of focal attention. Perception and Psychophysics 1986;45:225-240.

Eulitz C, Maess B, Pantev C, Friederici AD, Feige B, Elbert T. Oscillatory neuromagnetic activity induced by language and non-language stimuli. Cog Brain Res 1996;4:121-132.

Feige B. Oscillatory brain activity and its analysis on the basis of MEG and EEG, Münster: Westfälische Wilhelmsuniversität Münster, 1996.

Felleman DJ, van Essen DC. Distributed hierarchical processing in the primate cerebral cortex. Cerebral Cortex 1991;1:1-47.

Gomez-Gonzales CM, Clark VP, Fan S, Luck SJ, Hillyard SA. Sources of attention-sensitive visual event-related potentials. Brain Topog 1994;7(1):41-51.

Gray CM, Engel AK, König P, Singer W. Stimulus-dependent neuronal oscillations in cat visual cortex: receptive field properties and feature dependence. Eur J Neurosci 1990;2:607-619.

Haug AB, Baudewig J, Paulus W. Selective activation of human cortical area V5A by a rotating visual stimulus in fMRI; implication of attentional mechanisms. NeuroReport 1998;9:611-614.

Heilman KM. Attentional asymmetries, Cambridge: MIT-Press, 1995.

Heinze HJ, Mangun GR, Burchert W, Hinrichs H, Scholz M, Münte TF, Gös A, Scherg M, Johannes S, Hundeshagen H, Gazzaniga MS, Hillyard SA. Combined spatial and temporal imaging of brain activity during visual selective attention in humans. Nature 1994;372:543-546.

Hillyard SA, Anllo-Vento L. Event-related brain potentials in the study of visual selective attention. Proc Natl Acad Sci USA 1998;95:781-787.

Hillyard SA, Mangun GR, Woldorff MG, Luck SJ. Neural systems mediating selective attention. Cambridge: MIT Press 1995. pp. 665-681.

Hillyard SA, Vogel EK, Luck SJ. Sensory gain control (amplification) as a mechanism of selective attention: electrophysiological and neuroimaging evidence. Philosophical Transactions of the Royal Society Series B. 1998;353:1257-1270

Junghöfer M, Elbert T, Leiderer P, Berg P, Rockstroh B. Mapping EEGpotentials on the surface of the brain: a strategy for uncovering cortical sources. Brain Topography 1997;9:203-217.

Junghöfer M, Elbert T, Tucker DM, Rockstroh BS. Statistical control of artifacts in dense array EEG/MEG studies. Psychophysiology 1999; in press.

Keil A, Müller MM, Ray WJ, Gruber T, Elbert T. Human gamma band activity and perception of a gestalt. J Neurosci 1999;19:7152-7161.

Kirschfeld K. Oscillations in the insect brain: do they correspond to the cortical gamma-waves of vertebrates? Proc Natl Acad Sci USA. 1992;89:4764-4768.

LaBerge D. Attentional processing, Cambridge: Harvard University Press, 1995.

Law SK, Rohrbaugh JW, Adams CM, Eckardt MJ. Improving spatial and temporal resolution in evoked EEG responses using surface Laplacians. Electroenceph clin Neurophysiol 1993;88:309-322.

Livingstone M, Hubel D. Segregation of form, color, movement, and depth:anatomy, physiology, and perception. Science 1988;240:740 749.

Luck SJ, Ford MA. On the role of selective attention in visual perception. Proc Natl Acad Sci USA 1998;95:825-830.

Luck SJ, Chelazzi L, Hillyard SA, Desimone R. Neural mechanisms of spatial selective attention in areas V1. V2, and V4 of macaque visual cortex. J Neurophysiol 1997;77:24-42.

Lutzenberger W, Pulvermüller F, Elbert T, Birbaumer N. Visual stimulation alters local 40-Hz responses in humans: an EEG-study. Neurosci Lett 1995;183:39-42.

Makeig S. Auditory event-related dynamics of the EEG spectrum and effects of exposure to tones. Electroenceph clin Neurophysiol 1993;86:283-293.

Malsburg CVD, Schneider W. A neural cocktail-party processor. Biol Cybern 1986;54:29-40.

Mangun GR. Neural mechanisms of visual selective attention. Psychophysiology 1995;32:4-18.

Mangun GR, Hillyard SA, Luck SJ. Electrocortical substrates of visual selective attention. In: Meyer D, Kornblum S, editors. Attention and Performance XIV, Cambridge: MIT Press, 1993. pp. 219-243.

McCarthy G, Wood CC. Scalp distributions of event-related potentials:an ambiguity associated with analysis of variance models. Electroenceph clin Neurophysiol 1985;62:203-208.

Milner PM. A model for visual shape recognition. Psychological Review 1974;81(6):521-535.

Müller MM. Oscillatory cortical activities in the human brain. Habilitation, University of Konstanz. Electronic publication, University of Konstanz, http://www.uni-konstanz.de/ZE/Bib/habil/muellermc/muehab.htm, 1998

Müller MM, Bosch J, Elbert T, Kreiter A. Valdes Sosa. M., Valdes Sosa, P., Rockstroh, B. Visually induced gamma-band responses in human electroencephalographic activity - a link to animal studies. Exp Brain Res 1996;112:96-102.

Müller MM, Elbert T, Rockstroh B. Visuell induzierte Gammaband-aktivität im menschlichen EEG - Ausdruck corticaler Reizrepräsentation? Zeitschrift für Experimentelle Psychologie 1997a;44:186-212.

Müller MM, Junghöfer M, Elbert T, Rockstroh B. Visually induced gamma-band responses to coherent and incoherent motion: a replication study. NeuroReport 1997b;8:2575-2579.

Müller MM, Teder-Sälejärvi W, Hillyard SA. The time course of cortical facilitation during cued shifts of spatial attention. Nature Neurosci 1998;1:631-634.

Neshige R, Lüders H, Shibasaki H. Recording of movement-related potentials from scalp and cortex in man. Brain 1988;111:719-736.

Nunez PL. Localization of brain activity with electroencephalography. In: Sato S, editor. Magnetoencephalography, 54. New York: Raven Press, 1990. pp. 39-65.

Ota T, Toyoshima R, Yamauchi T. Measurements by biphasic changes of the alpha band amplitude as indicators of arousal level. Int J Psychophysiol 1996;24:25-37.

Palmer T, Tzeng OJL. Cerebral asymmetry in visual attention. Brain and Cognition 1990;13:46-58.

Patzwahl DR, Elbert T, Zanker JM, Altenmüller EO. The cortical repre- 
sentation of object motion in man is interindividually variable. NeuroReport 1996;7:469-472.

Perrin F, Pernier J, Bertrand O, Echallier JF. Spherical splines for scalp potential and current source density mapping. Electroenceph clin Neurophysiol 1989;72:184-187.

Posner MI, Dehaene S. Attentional networks. TINS. 1994;17:75-79.

Posner IP, Petersen SE. The attention system of the human brain. Annu Rev Neurosci 1990;13:25-42.

Posner MI, Snyder CRR, Davidson BJ. Attention and detection of signals. J Exp Psychol :General 1980;109:160-174.

Posner MI, Inhoff AW, Friedrich FJ, Cohen A. Isolating attentional systems: a cognitive-anatomical analysis. Psychobiology 1987; 15:107-121.

Priestley MB. Non-linear and non-stationary time series analysis, London: Academic Press, 1988.

Probst T, Plendl H, Paulus W, Wist ER, Scherg M. Identification of the visual motion area (area V5) in the human brain by dipole sorce analysis. Exp Brain Res 1993;93:345-351.

Pulvermüller F. Hebb's concept of cell assemblies and the psychophysiology of word processing. Psychophysiology 1996;33:317-333.

Pulvermüller F, Preissl H, Lutzenberger W, Birbaumer N. Brain rhythms of language: nouns versus verbs. Eur J Neurosci 1996;8:937-941.

Pulvermüller F, Birbaumer N, Lutzenberger W, Mohr B. High-frequency brain activity:its possible role in attention, perception and language processing. Prog Neurobiol 1997;52:427-444.

Rougeul-Buser A, Buser B. Electrocortical rhythms in the attentive cat: Phenomenological data and theoretical issues. In: Pantev C, Elbert T, Lutkenhöner B, editors. Oscillatory event related brain dynamics, New York, London: Plenum Press, 1994.

Sereno MI, Dale AM, Reppas JB, Kwong KK, Belliveau JW, Brady TJ, Rosen BR, Tootell RBH. Borders of multipe visual areas in humans revealed by functional magnetic resonance imaging. Science 1995;268:889-893.

Sheer DE. Sensory and cognitive $40 \mathrm{~Hz}$ event-related potentials: behavioral correlates, brain function, and clinical application. In: Basar E, Bullock $\mathrm{TH}$, editors. Brain dynamics: progress and perspectives, 2. Berlin: Springer-Verlag, 1989. pp. 339-374.

Singer W, Gray CM. Visual feature integration and the temporal correlation hypothesis. Ann Rev Neurosci 1995;18:555-586.

Singer W, Gray C, Engel A, König P, Artola A, Bröcher S. Formation of cortical cell assemblies. Cold Spring Harbor Symposia on Quantitative Biology. 1990;LV:939-952.

Tallon C, Bertrand O, Bouchet P, Pernier J. Gamma-range activity evoked by coherent visual stimuli in humans. Eur J Neurosci 1995;7:12851291.

Tallon-Baudry C, Bertrand O, Delpuech C, Pernier J. Stimulus specificity of phase-locked and non-phase-locked $40 \mathrm{~Hz}$ visual response in human. J Neurosci 1996;16(13):4240-4249.

Tallon-Baudry C, Bertrand O, Delpuech C, Pernier J. Oscillatory gammaband $(30-70 \mathrm{~Hz})$ activity induced by a visual search task in human. J Neurosci 1997a;17:722-734.

Tallon-Baudry C, Bertrand O, Wienbruch C, Ross B, Pantev C. Combined EEG and MEG recordings of visual $40-\mathrm{Hz}$ responses to illusory triangles in human. NeuroReport 1997b;8:1103-1107.

Tallon-Baudry C, Bertrand O, Peronnet F, Pernier J. Induced gamma-band activity during the delay of a visual short-term memory task in humans. J Neurosci 1998;18:4244-4254.

Tomberg C, Noel P, Ozaki I, Desmedt JE. Inadequacy of the average reference for the topographic mapping of focal enhancements of brain potentials. Electroenceph clin Neurophysiol 1990;77:259-265.

Tootell RBH, Reppas JB, Dale AM, Look RB, Sereno MI, Malach R, Brady TJ, Rosen BR. Visual motion aftereffect in human cortical area MT revealed by functional magnetic resonance imaging. Nature 1995;375:139-141.

Tootell RBH, Dale AM, Sereno MI, Malach R. New images from human visual cortex. Trends Neurosci 1996;19:481-489.

Treisman AM. The perception of features and objects. In: Baddeley AD, Weiskrantz L, editors. Attention: selection, awareness, and control:a tribute to Donald Broadbent, Oxford, England: Clarendon Press/Oxford University Press, 1993. pp. 5-35.

Treisman AM, Gelade G. A feature-integration theory of attention. Cognitive Psychology 1980;12:97-136.

Treisman AM, Gormican S. Feature analysis in early vision: evidence from search asymmetries. Psychological Review 1988;95:15-48.

Ungerleider LG. Functional brain imaging studies of cortical mechanisms for memory. Science 1995;270:769-775.

Ungerleider LG, Haxby JV. 'What' and 'where'in the human brain. Current Opinion Neurobiol 1994;4:157-165.

Valdes-Sosa M, Bobes MA, Rodrigues V, Pinilla T. Switching attention without shifting the spotlight: object-based attentional modulation of brain potentials. J Cognitive Neurosci 1998;10:137-151.

Vijn PMC. Coherent neuronal activity underlying the EEG, University of Amsterdam, 1992.

Vijn PCM, van Dijk BW, Spekreijse H. Topography of occipital EEG reduction upon visual stimulation. Brain Topog 1992;5:117-181.

Zeki S. A Vision of the Brain. Oxford: Blackwell 1993. pp. 366. 